\title{
The mathematical work of $Y$. Matsushima and its development
}

\author{
Shoshichi KoBAYASHI*)
}

In the past thirty years, differential geometry has undergone an enormous change with infusion of topology, Lie theory, complex analysis, algebraic geometry and partial differential equations. Professor Matsushima played a leading role in this transformation by bringing new techniques of Lie groups and Lie algebras into the study of real and complex manifolds. He was a man of great insight; the problems he has worked on and the results he has obtained continue to be sources of inspiration for us geometers. When I was asked by the editorial board of the journal to write about Matsushima's work, I decided therefore to contribute something that would serve as a guide for students of geometry who wish to work in the area explored by him. I only hope that I am not leading them astray by committing too many errors.

In relating other papers to Matsushima's work, I tried, whenever possible, to cite for reference books and survey articles from which unquoted original papers can be easily traced back. I am grateful to Professors S. Murakami and K. Nomizu for calling my attention to several papers I overlooked in my first draft.

\section{Lie theory}

(1) Lie algebras and Lie groups, [a, 1, 3, 4, 5, 6, 7, 8, 9, 10].

Upon his graduation from the Osaka Imperial University in the fall of 1942, Matsushima became an Assistant in the Mathematical Institute of the newly established Nagoya Imperial University. Beginning with his first paper [1] in which he disproved a conjecture of Zassenhaus (that every semisimple Lie algebra $L$ of prime characteristic with $L=[L, L]$ is a direct sum of simple ideals), he spent the next ten years working on Lie groups and Lie algebras. During and also for several years after the Second World War, it was extremely difficult for Japanese mathematicians to gain access to foreign periodicals or to have their papers published. In [4], unaware of Chevalley's paper [1] Matsushima gave a separate algebraic proof on conjugacy of Cartan sublagebras. In [3] written with the knowledge of Chevalley's paper [2] only through the Mathematiacl Reviews, Matsushima gave new proofs to Chevalley's main results on replicas of matrices. (One can surmise adverse conditions at that time from the fact that the Proceedings of Japan Academy, vol. 23 (1947), containing

\footnotetext{
*) Partially supported by NSF Grant MCS82-00235 and SFB in Bonn.
} 
Matsushima's papers [3] and [4], consisted of only one issue of 156 pages, appeared in 1950 with a delay of three yaers). In 1946 the Physico-Mathematical Society of Japan evolved into two separate societies - the Mathematical Society of Japan and the Physical Society of Japan. The first volume of the Journal of the Mathematical Society of Japan contains three papers [5], [6], [7] of Matsushima. In [5], in addition to giving independent proofs to Chevalley-Tuan's [1] characerizations of linear algebraic Lie algebras, he characterizes the Lie group of an abstract algebraic Lie algebra. This work is closely related to Goto's paper [1] in the same issue of the Journal. (Goto was also at Nagoya at the time).

Matsushima's contributions to geometry of homogenoeus spaces began with his papers [8] and [9]. Montgomery and Samelson [1] and Borel [1] determined all connected compact Lie groups which act transitively on spheres. In [8], Matsushima strengthenẹd their results by determining (up to a small, finite number of cases) all connected compact Lie groups which act transitively on odd dimensional homology spheres.

In 1949, Malcev [1] obtained the following structure theorem on nilmanifolds $G / H$, i.e. quotients of nilpotent Lie groups $G$. (1) Every nilmanifold is homeomorphic to the product of a compact nilmanifold and a Euclidean space; (2) Every compact nilmanifold can be written as $G / D$, where $G$ is a simply connected nilpotent rational Lie group and $D$ is a discrete subgroup of $G$; (3) Two compact nilmanifolds with isomorphic fundamental groups are homeomorphic. Given a simply connected nilpotent Lie group $G$ and a discrete subgroup $D$, Matsushima [9] constructed a well adapted basis for the Lie algebra of $G$. Using this basis, he derived Malcev's structure theorem and proved, in addition, that, for $i=1,2$, the $i$-th cohomology $H^{i}(G / D ; \boldsymbol{R})$ of a compact nilmanifold $G / D$ (which is nothing but the cohomology $H^{i}(D ; \boldsymbol{R})$ of the group $D)$ is isomorphic to the Lie algebra cohomology $H^{i}(\mathrm{~g} ; \boldsymbol{R})$ of ChevalleyEilenberg. A few years later, Nomizu [1] extended this isomorphism to all $i$. These results of Malcev and Matsushima have more or less closed the chapter on nilmanifolds and motivated Mostow, H.C. Wang, L. Auslander and others to investigate the structure of solvmanifolds (see Auslander [1]). In his dissertation under Matsushima, Sakane [1] obtained the $\bar{\partial}$ analogue of the isomorphism theorem of Matsushima-Nomizu for compact complex nilmanifolds. For certain compact complex solvmanifolds $G / D$, he obtained also an isomorphism theorem for $H^{0,1}(G / D)$.

Matsushima's book [a] on the theory of Lie algebras, published several years before Jacobson's, played a major role in Japan in making the theory readily accessible to non-experts.

(2) Lie pseudogroups and differential systems, [11, 12, 13, 14].

Chern, Ehresmann, Libermann and Matsushima were the first to reexamine the work of E. Cartan and Kähler on exterior differential systems. Cartan 
proved that the prolonged system of an exteiror differential system in involution is also in involution when the system consists of forms of degree less than or equal to two. In [11], Matsushima extended this result to an arbitrary differential system. This work and his seminar on differential systems at Nagoya University in 1952/53 introduced Kuranishi to the subject and motivated him to prove his famous prolongation theorem, (Kuranishi [1]). (Reminiscing his years in Nagoya, Matsushima wrote once that, although he himself got relatively little out of differential systems for the time and effort spent, he felt much gratified with Kuranishi's great success).

Chevalley, then at Columbia, spent the fall of 1953 in Nagoya. He was the first foreign mathematician to visit Japan after the war for an extended period of time. Matsushima spoke of Chevalley's three months in Nagoya as an event which left upon him a deep and lasting impression. In 1954, he was invited by Chevalley and $\mathrm{H}$. Cartan to France as a member of CNRS. Interested in differential systems and Lie pseudogroups, he spent the first six months in Strasbourg. In his paper [13] presented at Ehresmann's seminar in Strasbourg, he extended E. Cartan's classification of complex irreducible infinite Lie algebra to the real case. It was ten years later that irreducible and, more generally, primitive infinite Lie algebras began to attract many other mathematicians. The development of this subject between 1963 and 1969 culminating in the classification of primitive infinite Lie algebras, simplifying and completing the work of Cartan, can be easily traced back from the papers of Guillemin [1] and Morimoto-Tanaka [1].

In the spring of 1955, Matsushima left Strasbourg for Paris and lectured on infinite Lie pseudogroups at Bourbaki Seminar [14]. This lecture which placed the theory of Lie pseudogroups on a solid footing using Ehresmann's theory of jets has become a frequent source of reference on the subject.

\section{Homogeneous complex manifolds}

(1) Homogeneous Kähler manifolds, [15, 16, 17, 22].

Matsushima returned to Nagoya in December, 1955. His sojourn in France seems to have determined the course of his research for the next several years.

In 1954 there appeared two papers of H.C. Wang [1], [2], in which the structure of simply connected compact homogeneous complex manifolds and that of holomorphically parallelizable complex manifolds were determined. The problem of extending Wang's results to a wider class of homogeneous complex manifolds was an important problem. Another problem which attracted many geometers at that time was to prove that every bounded homogeneous domain in $\boldsymbol{C}^{n}$ is symmetric. (This had been proven for $n \leqq 3$ by E. Cartan who classified all symmetric bounded domains in 1935).

Using an interplay between holonomy and isotropy of a homogeneous 
Riemannian manifold, Lichnerowicz [1] proved in 1952 that a homogeneous Kähler manifold $M=G / H$ with nonzero Ricci tensor (e.g. a bounded homogeneous domain with its Bergman metric) is symmetric if the linear isotropy representation of $H$ is irreducible. In another paper (Lichnerowicz [2]), this was extended to the Hermitian case. While in Paris, Matsushima [15] generalized these results of Lichnerowicz by showing that a homogeneous complex manifold $M=G / H$ with $H$ compact is symmetric if the linear isotropy representation of $H$ is irreducible, thus removing the assumption on the Ricci tensor (or on the holonomy).

The Ricci tensor of a Kähler manifold can be obtained by applying $\partial \bar{\partial} \log$ to the volume form. In 1954, Koszul [1] studied the class of homogeneous complex manifolds $M=G / H$ (not necessarily Kähler) with an invariant volume form such that the associated Ricci tensor is non-degenerate. He proved that if an $n$-dimensional homogeneous complex manifold $M=G / H$ with $G$ semisimple and $H$ compact has non-degenerate Ricci tensor of signature, say $k$ pluses and $n-k$ minuses, then the dimension of the maximal compact subgroup $K$ is equal to $k+\operatorname{dim} H$. From this he obtained the theorem that a homogeneous bounded domain $M=G / H$ with $G$ semisimple is symmetric. This latter result was obtained independently also by Borel [2] who proved that if $M=G / H$ is a homogeneous Kähler manifold with $G$ semisimple and if $K \supset H$ is a maximal compact subgroup of $G$, then $G / H$ is a holomorphic fibre bundle over a bounded symmetric domain $G / K$ with a compact homogeneous Kähler manifold $K / H$ as fibre. This was generalized by Matsushima [16] to the case where $G$ is reductive. Namely, he proved that if $M=G / H$ is a homogeneous Kähler manifold with $G$ reductive and if $S$ is the semisimple part of $G$ and $C$ is the center of $G$, then $M$ is a Kählerian direct product of $S / H$ and $C$. This has two consequences; (i) A bounded homogeneous domain $M=G / H$ with $G$ reductive is symmetric and (ii) every compact homogeneous Kähler manifold is a Kählerian direct product of a complex torus and a homogeneous Kähler manifold of a compact semisimple Lie group. This kindled the interest of Hano (also at Nagoya at the time) in the subject. Hano [1] proved that if $M=G / H$ is a homogenoeus Kähler manifold with $G$ unimodular and non-degenerate Ricci tensor, then $G$ is semisimple. As a consequence, every homogeneous bounded domain $M=G / H$ with $G$ unimodular is symmetric. It turned out that this is the best possible result in the affirmative direction. In 1959, PyatetskiiShapiro produced examples of non-symmetric homogeneous bounded domains (in dimension 4 and 5) and later he developed a theory of Siegel domains and showed that every homogeneous bounded domain can be realized as a Siegel domain, (see Pyatetskii-Shapiro [1] and Kaneyuki [1] as general references on the subject of homogeneous bounded domains).

Let $M=G / H$ be a homogeneous complex manifold with an invariant volume 
form. In the aforementioned papers, Koszul and Hano considered the case where the associated Ricci form is non-degenerate. As shown in Hano-Kobayashi [1], if the Ricci form is degenerate, then there is a closed subgroup $L$ of $G$ with $H \subset L \subset G$ such that the integral submanifolds of the null-space distribution of the Ricci form are precisely the fibres of the bundle $M=G / H$ over $W=G / L$ with fibre $F=L / H$. The fibres are complex submanifolds of $M$ and the base $W=G / L$ is a sympletic manifold carrying (the projection of) the Ricci form of $M$, but $W$ need not be a complex manifold. In [22], Matsushima shows that if $M$ is compact with $G$-invariant volume and $G$ is semisimple (but not necessarily compact), then $W$ is a compact homogeneous Kähler manifold of a compact semisimple Lie group $G_{1}$, where $G_{1}$ is the compact component of $G$, and the fibre $F$ is the quotient of a reductive complex Lie group by a discrete subgroup. This generalizes Wang's results.

This fibration was extended to all compact homogeneous complex manifolds $M$ by Borel and Remmert [1]. Let $M=G / H$ where $G$ is a complex Lie group and $H$ is a closed complex Lie subgroup. Let $N$ be the normalizer of $H^{0}$ (the identity component of $H$ ) in $G$. Then $M$ is a holomorphic bundle over $W=$ $G / N$ with fibre $F=N / H=\left(N / H^{0}\right) /\left(H / H^{0}\right)$, and $W$ is a simply connected compact homogeneous Kähler manifold and $F$ is the quotient of a complxe Lie group $N / H^{0}$ by a discrete subgroup $H / H^{0}$. Using this fibration and also the Albanese fibration, they showed that if $M$ admits a Kähler metric (which may not be $G$-invariant), then $M$ is a direct product $W \times F$ and $F$ is a complex torus. The same result was obtained also by Tits [1]. This generalizes one of the results in Matsushima [16] mentioned above. A more function theoretic approach to this fibration can be found in Grauert-Remmert [1]. According to Remmert and van den Ven (see Potters [1]), the Albanese fibration exists for any compact almost homogeneous complex manifold. Tits [1] classified all compact homogeneous complex manifolds of dimension $\leqq 3$, and Potters [1], classified all compact almost homogeneous complex surfaces. Huckleberry and Livorni [1], former colleagues of Matsushima at Notre Dame, obtained the classification of homogeneous noncompact complex surfaces of complex Lie groups. Their proof makes use of results in Matsushima [19] and [21].

The outstanding problem in this area is the fundamental conjecture of Vinberg and Gindikin [1] which states that every homoegneous Kähler manifold is a holomorphic fibre bundle whose base is a homogeneous bounded domain and whose fibre is the direct product of a flat homogeneous Kähler manifold and a simply connected compact homogeneous Kähler manifold. For recent progress on this, see Shima [1] and Dorfmeister [1].

(2) Homogeneous Stein manifolds, [20, 21, 23].

One of the central problems in several complex variables has always been geometric and function theoretic characterizations and constructions of Stein 
manifolds. At the Brussel conference on several complex variables in 1953, $\mathrm{J}-\mathrm{P}$. Serre asked the following question. If $E$ is a holomorphic fibre space whose base and fibre are both Stein manifolds, is it also a Stein manifold? He knew then the answer to be affirmative for any holomorphic vector bundle $E$ or for any holomorphic principal bundle $E$ whose structure group is a closed complex Lie subgroup of $G L(m ; \boldsymbol{C})$. In 1955, Stein showed that any unramified covering manifold of a Stein manifold is again a Stein manifold, thus answering Serre's question when the fibre is discrete. In [20], Matsushima and Morimoto gave an affirmative answer in a very important case, namely when the structure group of $E$ is a connected complex Lie group. By Oka's principle (proved by Grauert) and Lie theory, they reduced the proof to the case when $E$ is a principal bundle. In the process, they proved the following results which are of independent interest. (i) A complex Lie group acting on a Stein manifold is Stein. (ii) A connected complex Lie group is Stein if and only if no complex Lie subgroup is contained in a compact subgroup. (iii) If a holomorphic principal bundle is Stein and if its structure group is the complexification of a maximal compact subgroup, then the base is Stein.

These results have led Matsushima naturally to his papers [21] and [23] on homogeneous Stein manifolds. The result (iii) above applies to the case where the total space of a principal bundle is a Stein group $G$ and the structure group is a connected closed complex Lie subgroup $H$ so that the base is the homogeneous complex manifold $G / H$. In [21], Matsushima proved the converse of (iii) in this case assuming that $G$ is semisimple. Thus, for a connected complex semisimple Lie group $G$ and a connected closed complex Lie subgroup $H$, the quotient space $G / H$ is Stein if and only if $H$ is the complexification of a maximal compact subgroup (of $H$ ). In [23], he treated the general case where $G$ is not necessarily semisimple.

Morimoto, Kopfermann, Villani, Kazama and S. Takeuchi continued this line of investigation. I quote one result from Takeuchi [1] which contains other references on this subject. Among other things, he proves that if $P$ is a holomorphic principal bundle over a $q$-complete manifold with a connected $r$-complete structure group $G$, then $P$ is $(q+r)$-complete. (" $q$-complete" in his definition is " $(\mathrm{n}-q+1)$-complete" in the sense of Andreotti-Grauert, where $n$ is the dimension of the manifold, so that " $n$-complete" is "Stein condition").

After the work of Matsushima and Morimoto, people turned their attention to the case where the fibre is a bounded pseudoconvex domain (for which the automorphism group is a real Lie group). In this connection, G. Fischer introduced the concept of Banach-Stein manifold and gave the affirmative answer to Serre's question when the fibre is Banach-Stein. Stehle introduced the concept of hyperconvex manifold also to answer positively the question of Serre when the fibre is hyperconvex. Many others made contributions to this prob- 
lem by giving sufficient conditions for a Stein manifold to be either BanachStein or hyperconvex. However, in 1977 Skoda constructed a holomorphic fibre space over an open set in $\boldsymbol{C}$ with fibre $\boldsymbol{C}^{2}$ such that every plurisubharmonic function is constant on each fibre, thus giving a negative answer to Serre's question. (It is essential that the automorphism group of $\boldsymbol{C}^{2}$ is very large). A simpler example (i.e., a simpler base space, a simpler structure group but with the same fibre $\boldsymbol{C}^{2}$ ) was later given by Demailly. For all these, see Pourcin's Bourbaki report [1] and Siu's survey article [1].

(3) Schubert varieties, [44].

This is one of Matsushima's last papers and answers a question raised by W. Stoll, a colleague of his at Notre Dame, in connection with multidimensional value distribution theory. Consider the following setting familiar in integral geometry. Let $S$ be a manifold fibered over two manifolds $F$ and $G$ with projections $\alpha: S \rightarrow F$ and $\beta: S \rightarrow G$. Given a differential form $\omega$ on $F$, we pull it back to a form $\alpha^{*} \omega$ on $S$ and then pull it down to a form $\beta_{*} \alpha^{*} \omega$ on $G$ by fiberintegration. The problem is to obtain a formula for $\beta_{*} \alpha^{*} \omega$ in the following concrete case. Fixing a $p$-tuple of non-negative integers $A=\left\{a_{1}, \cdots, a_{p}\right\}$, let $F$ be the space of flags $f$ of type $A$ in $\boldsymbol{C}^{m}$; a flag $f$ consists of subspaces $V_{1} \subset V_{2} \subset$ $\cdots V_{p}$ of $\boldsymbol{C}^{m}$ such that $\operatorname{dim} V_{f}=j+a_{f}$. Let $G$ be the Grassmann manifolds of $p$-planes in $\boldsymbol{C}^{m}$. For each flag $f$, let $S_{f}$ be the Schubert variety consisting of $p$-planes $V \in G$ such that $\operatorname{dim}\left(V \cap V_{j}\right) \geqq j$ for $j=1, \cdots, p$. Then set $S=\cup S_{f} \subset$ $F \times G$. Matsushima calculated $\beta_{*} \alpha^{*} \omega$ when $\omega$ is an invariant form of degree $(q, q)$ on $F$ using Kostant's results on Schubert varieties. Stoll's monograph [1] contains a more direct proof of this result of Matsushima and other related theorems.

\section{Vector bundle valued harmonic forms}

(1) Betti numbers of locally symmetric spaces, [24, 25, 28, 31].

In 1960, Matsushima returned to his Alma Mater, Osaka University, to fill the Chair of Algebra vacated by his teacher, Professor Shoda. It was in Osaka that he wrote a series of important papers on cohomology of locally symmetric spaces.

According to Kodaira's theory of deformations, the space of infinitesimal deformations of a pseudogroup structure is given in terms of the first cohomology with coefficients in the sheaf of germs of infinitesimal automorphisms. On the other hand, this cohomology group can be represented by harmonic forms by the Hodge theory. Using Bochner's technique of proving vanishing theorems, Calabi and Vesentini [1] obtained vanishing of certain cohomology groups $H^{q}(M, \Theta)$ for compact quotients $M$ of symmetric bounded domains and their sheaves $\Theta$ of germs of holomorphic vector fields. Using the same idea, Weil 
[1] proved the infinitesimal rigidity for uniform discrete subgroups of semisimple Lie groups.

In [24], Matsushima proved that if $M$ is a compact quotient of a symmetric bounded domain $X$ none of whose irreducible factors is equivalent to the unit ball in $\boldsymbol{C}^{m}$, then the first cohomology $H^{1}(M, \boldsymbol{R})$ vanishes. This brought out in striking contrast the difference between the classical 1-dimensional case and the higher dimensional case. As in the work of Calabi-Vesentini and Weil, positivity of a certain quadratic form defined by the curvature is the essential point of the proof.

In [25], Matsushima considered higher dimensional cohomology $H^{q}(M ; \boldsymbol{R})$. If $X^{*}$ is a symmetric space of compact type, then $H^{q}\left(X^{*} ; \boldsymbol{R}\right)$ or the space of harmonic $q$-forms on $X^{*}$ is given, according to E. Cartan, by invariant parallel $q$-forms on $X^{*}$. These $q$-forms induce invariant parallel $q$-forms on the noncompact dual $X$ which, in turn, induce parallel (and hence, harmonic) $q$-forms on the quotient $M$. Hence, $\operatorname{dim} H^{q}(M ; \boldsymbol{R}) \geqq \operatorname{dim} H^{q}\left(X^{*} ; \boldsymbol{R}\right)$ in general. Considering again positivity of a similar quadratic form, Matsushima determined the range of $q$ for which the equality $H^{q}(M ; \boldsymbol{R})=H^{q}\left(X^{*} ; \boldsymbol{R}\right)$ holds for each symmetric bounded domain $X$. Kaneyuki and Nagano [1], [2] completed Matsushima's program by treating the remaining symmetric spaces $X$ of noncompact type. Matsushima's lecture at the Bombay Colloquium [28] is a survey of his and their results on Betti numbers of locally symmetric spaces.

Matsushima's paper [31] which relates Betti numbers of locally symmetric spaces to unitary representations is of growing importance. Let $X=G / K$ be a symmetric space of noncompact type and $M=\Gamma \backslash X$ a compact locally symmetric space. In [31] he gave a formula for the Betti numbers of $M$ in terms of the multiplicities of certain unitary representations of $G$ in the right regular representation of $G$ on $L^{2}(\Gamma \backslash G)$. Hotta and Wallach [1] investiagte existence and non-existence of unitary representations appearing in the Matsushima formula and explain vanishing theorems in Matsushima's earlier papers [24], [25].

(2) Cohomology of vector bundles over locally symmetric spaces, [26, 27, 29, 30, 33].

In Osaka, Matsushima began a long and fruitful collaboration with Murakami on cohomology of vector bundles $E$ over compact locally symmetric spaces $M=\Gamma \backslash X$, where $X=G \mid K$ as before. Matsushima and Murakami considered two types of vector bundles. If a vector bundle $E$ is locally flat (i.e., transition functions are constant), the ordinary exterior differentiation $d$ can be defined for differential forms with values in $E$. In particular, if $E$ is induced by (the restriction to $\Gamma$ of) a representation $\rho$ of $G$, the de Rham cohomology of $M$ with values in $E$ can be defined. This will be denoted by $H^{p}(\Gamma, X, \rho)$. Similarly, if $E$ is holomorphic (so that it is "locally flat" with respect to $d$ ", i.e., $d$ " annihilates the transition functions), the $d^{\prime \prime}$-cohomology or the Dolbeault cohomology with values in 
$E$ can be defined. In particular, if $X$ is Hermitian symmetric and if $E$ is defined by the so-called canonical automorphic factor $J_{\rho}$ defined by a representation $\rho$ of $K$, this $d^{\prime \prime}$-cohomollogy is denoted by $H^{p, q}\left(\Gamma, X, J_{\rho}\right)$. When $\rho$ is the trivial representation, $H^{p}(\Gamma, X, \rho)$ reduces to the ordinary de Rham cohomology considered in [24], [25], [31], while $H^{1}(\Gamma, X, \rho)$ with $\rho=$ (adjoint representation) is the case considered by Calabi and Weil. On the other hand, $H^{p}(M, \Theta)$, where $\Theta=\mathcal{O}(T M)$, considered by Calabi-Vesentini is nothing but $H^{0, p}\left(\Gamma, X, J_{\rho}\right)$ where $\rho$ is the linear isotropy representation of $K$. In [26] and [29] Matsushima and Murakami studied cohomology groups $H^{p}(\Gamma, X, \rho)$ and $H^{p, q}\left(\Gamma, X, J_{\rho}\right)$ using harmonic theory for vector bundle valued forms and proved vanishing theorems generalizing and unifying earlier works of Calabi, Vesentini, Weil, Matsushima and Ise. Establishing a natural injection of $H^{p, 0}(\Gamma, X, \rho)$ into $H^{p, 0}\left(\Gamma, X, J_{\rho_{m}}\right)$, (where $\rho_{m}$ is a certain representation of $K$ determined by $\rho$ ), they proved that $H^{N, 0}(\Gamma, X, \rho),(N=\operatorname{dim} X)$, is isomorphic to the space of automorphhic forms with automorphic factor $J_{\tau}$, where $\tau$ is a certain representation determined by $\rho$, thus generalizing a theorem of Eichler and Shimura to higher dimensions.

Together with K. Nomizu of Brown University and K. Yano of Tokyo Institute of Technology, Matsushima organized the United States-Japan Seminar in Differential Geometry which was held in Kyoto in June, 1965; it was the first seminar sponsored by the U.S.-Japan Cooperative Science Program. The lecture [30] given at this seminar summarizes the results in [26] and [29]. See also Murakami [1, 2].

In [27] Matsushima and Shimura applied the techniques developed in [26] to the case where $X$ is the product of $n$ copies of the upper half-plane and obtained the explicit formulas for $\operatorname{dim} H^{p, q}(\Gamma, X, \rho)$ and, in particular, for the Betti numbers of the compact qoutient $\Gamma \backslash X$.

Matsushima's collaboration with Murakami continued even after he moved to Notre Dame in 1966. As I mentioned above, in [31] Matsushima obtained an important formula expressing the Betti numbers of $M=\Gamma \backslash X=\Gamma \backslash G / K$ in terms of the multiplicities of certain unitary representations of $G$ on $L^{2}(\Gamma \backslash G)$. In [33] Matsushima and Murakami generalized this formula to express the dimension of the space $H^{q}\left(M, E\left(J_{\tau}\right)\right)$ of automorphic harmonic $q$-forms of type $J_{\tau}$ in terms of unitary representations of $G$.

For later developments of this subjects, i.e., vanishing theorem and the formula of Matusshima-Murakami, see the book of Raghunathan [1], the Seminar Notes by Borel and Wallach [1] and a survey lecture by Murakami [3]. A recent paper of Zucker [1] interpretes the results of Matsushima-Murakami from the standpoint of polarized variations of Hodge structures.

(3) Second fundamental forms and curvature forms, [37, 40].

Although [37] and [40] are on subjects entirely different from the papers quoted in (1) and (2), they use techniques of vector bundle valued harmonic 
forms. In studying minimal submanifolds it had been customary to apply the Laplacian to the length (squared) of the second fundamental form. In [37], in order to study submanifolds with parallel mean curvature normal in a space of constant curvature, Matsushima applied the Laplacian to the second fundamental form itself (considered as a vector bundle valued 1-form) and obtained a formula which includes those of Simons and others. Using the method of [37], Nomizu [2] proved the finite dimensionality of the space of generalized second fundamental forms and that of generalized curvature tensor fields for compact Riemannian manifolds.

In [40], Matsushima considered the curvature of a Kähler manifold as a vector bundle valued 2 -form and characterized the Einstein condition by its harmonicity. He obtained also an interesting inequality involving curvature components for a compact Kähler-Einstein manifold. Although the geometric meaning of the inequality has not been well understood, this is the first inequality of the kind involving more than the first and second Chern classes.

\section{Automorphisms of complex manifolds}

(1) Automorphisms of Siegel domains, [35, 36, 39].

In describing Matsushima's work on homogeneous Kähler manifolds, I mentioned the theory of Siegel domains developed by Pyatetskii-Shapiro. A tube domain over a convex cone such as a Siegel's upper half plane is an example of Siegel domain. In the joint work [35] with W. Kaup and T. Ochiai, Matsushima obtained structure theorems for the Lie algebra of the automorphism group of an arbitrary (not necessarily homogeneous) Siegel domain. In particular, this Lie algebra $\mathfrak{g}$ consists of polynomial vector fields and is graded in a natural way: $\mathfrak{g}=\mathfrak{g}_{-1}+\mathfrak{g}_{-1 / 2}+\mathfrak{g}_{0}+\mathfrak{g}_{1 / 2}+\mathfrak{g}_{1}$. They constructed also a natural equivariant imbedding of a Siegel domain $D$ into $P_{N} C$ as an open set of an algebraic variety of $P_{N} C$, where $N=\left(\begin{array}{c}r \\ n\end{array}\right)-1, r=\operatorname{dim} g$ and $n=\operatorname{dim} D$. Moreover, they answered affirmatively the conjecture of Pyatetskii-Shapiro that the realization of a domain as a Siegel domain is unique up to affine transformations. These results were announced in [36] at a meeting in Maryland. In his lecture [39] at Washington University, Matsushima explained the results of [35] and the theorem of Vey (that a Siegel domain which has a compact quotient must be symmetric) in the special case of tube domains.

Closely related structure theorems for the Lie algebra $g$ were obtained independently by Tanaka [1]. A unified exposition of all these results can be found in Murakami's lecture at Tata [4].

(2) First Chern class and automorphisms of compact Kähler manifolds, $[18,34,38, \mathrm{c}]$.

According to a theorem of Yano, every Killing vector field (i.e., an in- 
finitesimal isometry) of a compact Kähler manifold $M$ is holomorphic. In other words, the Lie algebra $\mathfrak{g}$ of Killing vector fields is a real Lie subalgebra of the Lie algebra $\mathfrak{a}$ of holomorphic vector fields. According to Bochner, $\mathfrak{a}=0$ if the Ricci tensor is negative while $\mathfrak{g}=\mathfrak{a}$ consists of parallel vector fields if the Ricci tensor is zero, (see Yano-Bochner [1]). In [18], Matsushima proved that if $M$ is a compact Einstein-Kähler manifold with positive Ricci tensor, then $\mathfrak{a}=\mathfrak{g}+i \mathfrak{g}$, i.e., $\mathfrak{a}$ is the complexification of $\mathfrak{g}$. Since the group $G$ of isometries is compact and its Lie algebra is reductive, it follows that $\mathfrak{a}$ is reductive. This is an important necessary condition for the existence of an Einstein-Kähler metric. Thus, for a compact complex manifold $M$ with positive first Chern class $c_{1}$ to admit an Einstein-Kähler metric, it is necessary that its Lie algebra $\mathfrak{a}$ of holomorphic vector fields is reductive. This has been the only known obstruction for the existence of an Einstein-Kähler metric until the recent discovery of another obstruction by Futaki [1]. (When $c_{1}<0$ or $c_{1}=0$, there is no such obstruction by the results of Aubin [1] and Yau [1], see also Seminaire Palaiseau [1]). This result of Matsushima was immediately generalized by Lichnerowicz [3] to the case of constant scalar curvature; if $M$ is a compact Kähler manifold with constant scalar curvature, then $\mathfrak{a}=\mathfrak{g}+i \mathfrak{g}$, with $\mathfrak{g} \cap i \mathfrak{g}$ consisting of parallel vector fields, and $\mathfrak{a}$ is reductive.

In the above quoted work and later papers, Lichnerowicz $[3,4,5]$ studied systematically the ideal $\mathfrak{i}$ of $\mathfrak{a}$ consisting of holomorphic vector fields which annihilate all holomorphic 1-forms by interior product operation. In [34] and [38], Matsushima considered $\mathfrak{i}$ as the Lie algebra of the group $I$ of holomorphic transformations of $M$ which induce the identity transformation on the Albanese variety $A(M)$. In this way, he obtained the following structure theorem for a Hodge manifold $M$ with $c_{1}=0$. A suitable finite regular covering space $M^{*}$ of $M$ with solvable covering transformation group can be written as the direct product $A \times F$ of an abelian variety $A$ and a Hodge manifold $F$ with $c_{1}(F)=0$ and irregularity $q=0$ (i.e., the first Betti number $b_{1}=0$ ). The same structure theorem for a compact Kähler manifold with zero Ricci tensor had been known to Calabi [1]. Owing to Yau's solution of the Calabi conjecture for $c_{1}=0$ and the work of Cheeger-Gromoll [1] and Fischer-Wolf [1], the structure theorem of Calabi and Matsushima holds now for any compact Kähler manifold $M$ with $c_{1}=0$ with a stronger conclusion that $F$ is simply connected, (see Kobayashi [1]).

Matsushima's CBMS note [c] is an excellent introduction to the subject described here. Some of the results of Matsushima and Lichnerowicz on holomorphic transformations of compact Kähler manifolds are explained also in Chapter III of Kobayashi's book [2]. Some results of Matsushima on holomorphic vector fields on Hodge manifolds have been generalized since then to the Kähler case by Carrell and Lieberman [1]. 


\section{Complex tori}

(1) Vector bundles over complex tori, $[19,45,46]$.

In 1957, the problem of classifying holomorphic vector bundles was solved in the two special cases: when the base space is the Riemann sphere (by Grothendieck) and when the base space is an elliptic curve (by Atiyah). At that time nothing was known for the classification of holomorphic vector bundles over higher dimensional base spaces. Matsushima's paper [19] may be called the first paper on stable vector bundles on complex tori of higher dimension. He proved that a holomorphic vector bundle $E$ over a complex torus $T$ admits a holomorphic connection if and only if it is homogeneous. He showed also that such a bundle comes from a representation of the fundamental group of $T$ if the rank of $E$ is 2 . This was immediately generalized to the case of arbitrary ranks by Morimoto [1]. For generalizations to arbitrary characteristics, see Umemura [1] and Miyanishi [1].

In [45], Matsushima studied those holomorphic vector bundles over a complex torus $T$ which come from representations of Heisenberg groups and essentially determined their structure by classifying the irreducible representations of the Heisenberg groups. In a companion paper, Hano [2] showed that the projective bundle $P(E)$ associated with such a vector bundle $E$ admits a holomorphic connection and comes from a representation of the fundamental group of $T$ into the projective general linear group. So, the bundles constructed by Matsushima are semi-homogeneous vector bundles in Mukai [1].

In [46], Matsushima considered complex tori $T$ with a Riemann form of signature $(s, r)$, where $s+r=n$ is the $\operatorname{dim} T$, in other words, with a line bundle $L$ whose Chern class, represented by an invariant $(1,1)$-form on $T$, is of signature $(s, r)$. To each $(T, L)$ he attaches a family of polarized abelian varities $\left(T_{b}, L_{b}\right)$ parametrized by elements $b$ of the symmetric domain $U(s, r) / U(s) \times U(r)$ (by decomposing the tangent spaces of $T$ into subspaces of dimensions $r$ and $s$ and reversing the complex structures of these $s$-dimensional subspaces) and showed that $H^{r}(T, L)$ is naturally isomorphic to $H^{0}\left(T_{b}, L_{b}\right)$. The same construction had been considered by Griffiths [1] in the context of comparing his Jacobians $T$ with Weil's Jacobians $T_{b}$.

(2) Ample bundles and subvarities of complex tori, [41, 42, 43].

Let $E$ be a holomorphic vector bundle over a compact complex manifold $M$ such that the fibres are generated by global sections. Such a bundle is called ample in [41] but weakly ample in [43] in order to avoid the conflict with the standard terminology. The most natural example of such a vector bundle is the cotangent bundle of a submanifold in a complex torus. In the joint work [41] with Stoll, Matsushima proved that if $E$ is a weakly ample vector bundle of rank $r$ such that its (refined) Chern classes $c_{1}, \cdots, c_{r}$ satisfy $c_{1}^{k_{1}} \cdots c_{r}^{k_{r}} \neq 0$ for some 
$\left(k_{1}, \cdots, k_{r}\right)$, then the transcendence degree of the field of meromorphic functions of $M$ is at least $\sum_{i=1}^{r} i k$. In particular, it was shown that if $E$ is a weakly ample vector bundle over a compact Kähler manifold and if one of the Chern numbers of $E$ is nonzero, then $M$ is projective algebraic. This may be considered as a generalization of the famous Kodaira imbedding theorem. This can be applied to the tangent bundle of a compact homogeneous complex manifold as well as to the cotangent bundle of a submanifold in a complex torus. In [42] Matsushima studied the structure of hypersurfaces $M$ in a complex torus $T$ of dimension $n+1$, or equivalently, compact Kähler manifolds $M$ of dimension $n$ with a weakly ample vector bundle $E$ having exactly $n+1$ linearly independent sections. He proved that such a manifold $M$ is a fibre bundle over $N=M / \mathrm{Aut}_{0} M$ with fibre $\mathrm{Aut}_{0} M$, where $\mathrm{Aut}_{0} M$ is the identity component of the automorphism group of $M$ and is also a complex torus. Moveover, $N$ is also a hypersurface of a complex torus and its Euler number is nonzero. In [43], Chern numbers of a compact Kähler surface immersed in a complex torus are studied. For the structure theorem for submanifolds (of arbitrary codimensions) in complex tori, see the paper of B. Smyth [1]. These papers of Matsushima, Stoll, Howard and Smyth played important roles in the resolution of the Bloch conjecture concerning holomorphic maps from the complex line $C$ into a submanifold of a complex torus. (see Ochiai [1] and P-M. Wong [1]). Matsushima's paper [42] is also closely related to the work of Nagano and Smyth [1] who considered, more generally, minimal submanifolds of flat tori.

(3) Affine structures, [32].

This is one of the earliest papers if not the earliest on the subject of holomorphic (flat) affine structures. Matsushima showed that the set of affine structures on a compact homogeneous complex manifold forms a complex affine variety. In particular, he proved that the set of affine structures on an $n$-dimensional complex torus is in a natural one-to-one correspondence with the set of commutative associative algebra structures on the vector space $\boldsymbol{C}^{n}$. According to Markowitz [1], an affine structure on a complex torus is projectively hyperbolic (in the sense of Kobayashi) if and only if the corresponding algebra is semisimple.

K. Yagi's thesis written at Notre Dame under the direction of Matsushima is on compact homogeneous (real) affine manifolds, (see Yagi [1]). See also Nagano and Yagi [1] for affine structures on real tori.

Vitter [1] and Sakane [2] continued the work of Matsushima on holomorphic affine structures. In the past ten years, the subject of holomorphic affine structures has beccme an active area of research, see, for example, Gunning's note [1] and Kobayashi-Wu [1] for other references. 


\section{References}

Aubin, T. [1], Equations du type Monge-Ampère sur les variétés kähleriennes compactes, C.R. Acad. Sci. Paris 283 (1976), 119-121.

Auslander, L. [1], On the theory of solvmanifolds and generalizations with applications to differential geometry, Proc. Symp. Pure Math. III, Amer. Math. Soc. 1961, 138143.

Borel, A. [1], Some remarks about Lie groups transitive on spheres and tori, Bull. Amer. Math. Soc. 55 (1949), 580-587.

Borel, A. [2], Kählerian coset spaces of semi-simple Lie groups, Proc. Nat. Acad. Sci. U.S.A. 40 (1954), 1147-1151.

Borel, A. and Remmert, R. [1], Über kompakte homogene Kählersche Mannigfaltigkeiten, Math. Ann. 145 (1961/62), 429-439.

Borel, A. and Wallach, N. [1], Continuous cohomology, discrete subgroups, and representations of reductive groups, Ann. Math. Studies, No. 94 (1980), Princeton Univ. Press.

Calabi, E. [1], On Kähler manifolds with vanishing canonical class, Algebraic Geometry \& Topology, Symp. in honor of S. Lefschetz, 78-89, Princeton, 1957.

Calabi, E. and Vesentini, E. [1], On compact, locally symmetric Kähler manifolds, Ann. of Math. 71 (1960), 472-507.

Carrell, J. and Liebermann, D. [1], Holomorphic vector fields and Kähler manifolds, Invent. Math. 21 (1973), 303-309.

Cheeger, J. and Gromoll, D. [1], The splitting theorem for manifolds of nonnegative Ricci curvature, J. Diff. Geometry 6 (1971), 119-128.

Chevalley, C. [1], An algebraic proof of a property of Lie groups, Amer. J. Math. 63 (1941), 785-793.

Chevalley, C. [2], A new kind of relationship between matrices, Amer. J. Math. 65 (1943), 521-531.

Chevalley, C. and Tuan, H.F. [1], On algebraic Lie algebras, Proc. Nat. Acad. Sci. U.S.A. 31 (1945), 195-196.

Dorfmeister, J. [1], Homogeneous Kähler manifolds admitting a transitive solvable group of automorphisms, to appear.

Fischer, A.E. and Wolf, J.A. [1], The structure of compact Ricci-flat Riemannian manifolds, J. Diff. Geometry 10 (1975), 277-288.

Futaki, A. [1], An obstruction to the existence of Einstein Kähler metrics, Invent. Math. 73 (1983), 437-443.

Goto, M. [1], On algebraic Lie algebras, J. Math. Soc. Japan 1 (1948), 29-45.

Grauert, H. and Remmert, R. [1], Über kompakte homogene komplexe Mannigfaltigkeiten, Arch. Math. 13 (1962), 498-507.

Griffiths, P.A. [1], Periods of integrals on algebraic manifolds, I, Amer. J. Math. 90 (1968), 568-626; II ibid. 805-865.

Guillemin, V. [1], Infinite dimensional primitive Lie algebras, J. Diff. Geometry 4 (1970), 257-282.

Gunning, R. [1], On uniformization of complex manifolds, the role of connections, Math. Note 22, Princeton Univ. Press, 1978.

Hano, J-I. [1], On Kaehlerian homogeneous spaces of unimodular Lie groups, Amer. J. Math. 79 (1957), 885-900. 
Hano, J-I. [2], A geometric characterization of a class of holomorphic vector bundles over a complex torus, Nagoya Math. J. 61 (1976), 197-202.

Hano, J-I. and Kobayashi, S. [1], A fibering of a class of homogeneous complex manifolds, Trans. Amer. Math. Soc. 94 (1960), 233-243.

Hotta, R. and Wallach, N. [1], On Matsushima's formula for the Betti numbers of a locally symmetric space, Osaka J. Math. 12 (1975), 419-431.

Huckleberry, A.T. and Livorni, E.L., [1], A classification of homogeneous surfaces, Canadian J. Math. 33 (1981), 1097-1110

Kaneyuki, S. [1], Homogeneous bounded domains and Siegel domains, Lecture Notes in Math. vol. 241 (1971), Springer-Verlag.

Kaneyuki, S. and Nagano, T. [1], On the first Betti numbers of compact quotient spaces of complex semi-simple Lie groups by discrete subgroups, Sci. Papers College General Educ. Univ. Tokyo 12 (1962), 1-11.

Kaneyuki, S. and Nagano, T. [2], On certain quadratic forms related to symmetric Riemannian spaces, Osaka Math. J. 14 (1962), 241-252.

Kobayashi, S. [1], Recent results in complex differential geometry, Jahresber. Deutsche Math.-Verein. 83 (1981), 147-158.

Kobayashi, S. [2], Transformation groups in differential geometry, Ergebnisse Math. Vol. 70, 1972, Springer-Verlag.

Kobayashi, S. and Wu, H. [1], Complex Differential Geometry, DMV Seminar, Vol. 3, 1983, Birkhauser.

Koszul, J-L. [1], Sur la forme hermitienne canonique des espaces homogènes complexes, Canadian J. Math. 7 (1955), 562-576.

Kuranishi, M. [1], On E. Cartan's prolongation theorem of exterior differential systems, Amer. J. Math. 79 (1957), 1-47.

Lichnerowicz, A. [1], Variétés pseudokählériennes à courbure de Ricci non nulle; application aux domaines bornés homogènes de $C^{n}$, C.R. Acad. Sci. Paris 235 (1952), 12-14.

Lichnerowicz, A. [2], Un théorème sur les espaces homogènes complexes, Arch. Math. 5 (1954), 207-215.

Lichnerowicz, A. [3], Isométries et transformations analytiques d'une variété kählérienne compacte, Bull. Soc. Math. France 87 (1959), 427-437.

Lichnerowicz, A. [4], Variétés kähleriennes et première classe de Chern, J. Diff. Geometry 1 (1967), 195-224.

Lichnerowicz, A. [5], Variétés kählériennes à première classe de Chern non-négative et variétés riemanniennes à courbure de Ricci generalisée non-négative, J. Diff. Geometry 6 (1971), 47-94.

Malcev, A. [1], On a class of homogeneous spaces, Amer. Math. Soc. Transl. No. 39 (1951),

Markowitz, M. [1], Holomorphic affine and projective connections of hyperbolic type, Math. Ann. 245 (1979), 55-62.

Miyanishi, M. [1], Some remarks on algebraic homogeneous vector bundles, Number Theory, Algebraic Geometry and Commutative Algebra, in honor of Y. Akizuki Kinokuniya, Tokyo (1973), 71-93.

Montgomery, D. and Samelson, H. [1], Transformation groups of spheres, Ann. of Math. 44 (1943), 454-470.

Morimoto, A. [1], Sur la classification des espaces fibrés vectoriels holomorphes sur 
xviii

un tore complexe admettant des connexions holomorphes, Nagoya Math. J. 15 (1959), 83-154.

Morimoto, T. and Tanaka, N. [1], The classification of the real primitive infinite Lie algebras, J. Math. Kyoto Univ. 10 (1970), 207-243.

Mukai, S. [1], Semi-homogeneous vector bundles on an abelian variety, J. Math. Kyoto Univ. 18 (1978), 239-272.

Murakami, S. [1], Cohomology of vector-valued forms on compact, locally symmetric Riemannian manifolds, Proc. Symp. Pure Math. vol. 9, Amer. Math. Soc. (1966), 387-399.

Murakami, S. [2], Cohomology groups of vector-valued forms on symmetric spaces, Lacture Notes, Univ. Chicago, 1966.

Murakami, S. [3], Certain cohomology groups attached to Hermitian symmetric spaces and unitary representations, Proc. Fifth General Meeting Southeast Asian Math. Soc., Hong Kong, 1980.

Murakami, S. [4], On automorphisms of Siegel domains, Lecture Notes in Math. 286, 1972, Springer-Verlag.

Nagano, T. and Smyth, B. [1], Minimal varieties and harmonic maps in tori, Comment. Math. Helv. 50 (1975), 249-265.

Nagano, T. and Yagi, K. [1], The affine structure on the real two-torus (1), Osaka J. Math. 11 (1974), 181-210.

Nomizu, K. [1], On the cohomology of compact homogeneous spaces of nilpotent Lie groups, Ann. of Math. 59 (1954), 531-538.

Nomizu, K. [2], On the spaces of generalized curvature tensor fields and second fundamental forms, Osaka J. Math. 8 (1971), 21-28.

Ochiai, T. [1], On holomorphic curves in algebraic varieties with ample irregularity, Invent. Math. 43 (1977), 83-96.

Potters, J. [1], On almost homogeneous compact complex analytic surfaces, Invent. Math. 8 (1969), 244-266.

Pourcin, G. [1], Fibrés holomorphes dont la base et la fibre sont des espaces de Stein, Sem. Bourbaki, Exposé 517 (1977/78), Lecture Notes in Math. No. 710,

Pyatetskii-Shapiro, I.I. [1], Automorphic functions and the geometry of classical domains, 1969, Gordon and Breach.

Raghunathan, M.S. [1], Discrete subgroups of Lie groups, Ergebnisse Math. vol. 68 (1972), Springer-Verlag.

Sakane, Y. [1], On compact complex parallelisable solvmanifolds, Osaka J. Math. 13 (1976), 187-212.

Sakane, Y. [2], On compact complex affine manifolds, J. Math. Soc. Japan 29 (1977), 135-149.

Séminaire Palaiseau [1], Première classe de Chern et courbure de Ricci; preuve de la conjecture de Calabi, Astérisque No. 58 (1978) Soc. Math. France.

Shima, H. [1], Homogeneous Kähler manifolds, Japanese J. Math., to appear.

Siu, Y-T. [1], Pseudoconvexity and the problem of Levi, Bull. Amer. Math. Soc. 84 (1978) 481-512.

Smyth, B. [1], Weakly ample Kähler manifolds and Euler number, Math. Ann. 224 (1976), 269-279.

Stoll, W. [1], Invariant forms on Grassmann manifolds, Ann. Math. Studies No. 89 (1977), Princeton Univ. Press. 
Takeuchi, S. [1], On completeness of holomorphic principal bundles, Nagoya Math. J. 56 (1975), 121-138.

Tanaka, N. [1], On the automorphisms of Siegel domains, J. Math. Soc. Japan 22 (1970), 180-217.

Tits, J. [1], Espaces homogènes complexes compacts, Comment. Math. Helv. 37 (1962), 111-120.

Umemura, H. [1], On a certain type of vector bundles over an abelian variety, Nagoya Math. J. 64 (1976), 31-45.

Vinberg, E.B. and Gindikin, S.G. [1], Kählerian manifolds admitting a transitive solvable automorphism group, Math. Sb. 74 (1967), 333-351.

Vitter, A. [1], Affine structures on compact complex manifolds, Invent. Math. 17 (1972), 231-244.

Wang, H-C. [1], Closed manifolds with homogeneous complex structure, Amer. J. Math. 76 (1954), 1-32.

Wang, H-C. [2], Complex parallisable manifolds, Proc. Amer. Math. Soc. 5 (1954), 771-776.

Weil, A. [1], On discrete subgroups of Lie groups II, Ann. of Math. 75 (1962), 578602.

Wong, P-M. [1], Holomorphic mappings into Abelian variety, Amer. J. Math. 102 (1980), 493-501.

Yagi, K. [1], On compact homogeneous affine manifolds, Osaka J. Math. 7 (1970), 457-475.

Yano, K. and Bochner, S. [1], Curvature and Betti numbers, Ann. Math. Studies, No. 32.

Yau, S-T. [1], On the Ricci curvature of a compact Kähler manifold and the complex Monge-Ampère equation I, Comm. Pure Appl. Math 31 (1978), 339-411.

Zucker, S. [1], Locally homogeneous variations of Hodge structure, L'Enseignment Math. 27 (1981), 243-276. 